\title{
Detection of Infarct Lesions From Single MRI Modality Using Inconsistency Between Voxel Intensity and Spatial Location-A 3-D Automatic Approach
}

\author{
Shan Shen, André J. Szameitat, and Annette Sterr
}

\begin{abstract}
Detection of infarct lesions using traditional segmentation methods is always problematic due to intensity similarity between lesions and normal tissues, so that multispectral MRI modalities were often employed for this purpose. However, the high costs of MRI scan and the severity of patient conditions restrict the collection of multiple images. Therefore, in this paper, a new 3-D automatic lesion detection approach was proposed, which required only a single type of anatomical MRI scan. It was developed on a theory that, when lesions were present, the voxel-intensitybased segmentation and the spatial-location-based tissue distribution should be inconsistent in the regions of lesions. The degree of this inconsistency was calculated, which indicated the likelihood of tissue abnormality. Lesions were identified when the inconsistency exceeded a defined threshold. In this approach, the intensity-based segmentation was implemented by the conventional fuzzy c-mean (FCM) algorithm, while the spatial location of tissues was provided by prior tissue probability maps. The use of simulated MRI lesions allowed us to quantitatively evaluate the performance of the proposed method, as the size and location of lesions were prespecified. The results showed that our method effectively detected lesions with 40-80\% signal reduction compared to normal tissues (similarity index $>0.7$ ). The capability of the proposed method in practice was also demonstrated on real infarct lesions from 15 stroke patients, where the lesions detected were in broad agreement with true lesions. Furthermore, a comparison to a statistical segmentation approach presented in the literature suggested that our 3-D lesion detection approach was more reliable. Future work will focus on adapting the current method to multiple sclerosis lesion detection.
\end{abstract}

Index Terms-Fuzzy c-mean (FCM), MRI, lesion detection, tissue probability map.

\section{INTRODUCTION}

$\mathbf{S}$ TROKE is one of the most common causes of death and the leading cause of severe disabilities in the U.K. [1], [2]. It occurs when an artery to the brain bursts or becomes blocked, thereby stopping or interrupting the blood supply to the brain [1]. This usually results in an infarct in the brain, which comprises dead tissues due to the stroke. The detailed information about the location and volume of the infarcts is crucial to a number of stroke-related research questions, such as predicting prognosis,

Manuscript received March 28, 2007; revised July 13, 2007 and October 2, 2007.

The authors are with the Department of Psychology, University of Surrey, Guildford GU2 7XH, U.K. (e-mail: shan.shen@surrey.ac.uk; a.szameitat@ surrey.ac.uk; a.sterr@surrey.ac.uk).

Color versions of one or more of the figures in this paper are available online at http://ieeexplore.ieee.org.

Digital Object Identifier 10.1109/TITB.2007.911310 identifying chronic functional deficits [3]-[7], or investigating sleep problems of stroke patients [8], [9]. In addition, the availability of a lesion mask may also be beneficial to analyses of functional MRI [10] and diffusion tensor imaging. Therefore, an effective lesion detection method is highly desirable.

With the fast advancement of MRI, which provides highresolution images, the detection of infarct lesion is becoming increasingly more feasible [11]. However, due to their arbitrary shapes and locations, segmentation of lesions is a complex and challenging task. Manual delineation is thus widely employed for this purpose [12], [13], but it is very time-consuming, which may be an unrealistic approach when a large amount of data needs to be processed. Another disadvantage of manual lesion delineation is that the results are highly dependent on the expertise of operators. In this case, continuous efforts have been made on automatic detection of different types of lesions in the literature. Although a great number of segmentation methods are available, such as thresholding [14], region growing [15], and clustering [16]-[21], they are problematic when applied to the lesion detection. This is because lesions often possess similar intensities to some normal tissues. For example, in T1weighted MR images, an infarct lesion is shown with intensities similar to those of cerebrospinal fluid (CSF) or gray matter (GM), so that a standard segmentation approach would group them together rather than separately. In order to solve this problem, multispectral MR images were employed for the purpose of lesion detection by many researchers. For example, Anbeek et al. [22]-[24] presented a segmentation method for white matter (WM) lesions using five different MRI modalities including T1-weighted, inversion recovery (IR), proton-density-weighted (PD), T2-weighted, and fluid attenuation inversion recovery (FLAIR). It is based on a K-nearest neighbor (KNN) classification technique, which builds a feature space from voxel intensity and spatial information. Sajja et al. [25] used PD-weighted, T2weighted, and FLAIR MR images to segment multiple sclerosis (MS) lesions, which involved techniques such as Parzen window classifier, morphological operations, hidden Markov random field-expectation maximization (HMRF-EM) algorithm, and fuzzy connectivity. A similar approach was employed by Datta et al. [26] to identify black holes in MS. Van Leemput et al. [27] proposed an intensity-based tissue classification algorithm implemented with T1-, T2-, and PD-weighted scans using a stochastic model for normal brain images and simultaneously detects MS lesions as outliers that are not well explained 
by the model. Many other lesion segmentation studies based on multispectral anatomical MRI scans were reported in [28]-[34].

However, in the majority of clinical situations, only one type of anatomical MR image is collected, since the acquisition of multispectral anatomical images is more time and cost intensive. Longer scanning times are further not feasible in many patients due to the severity of their condition. There is a large number of existing patient data with just one anatomical MRI. Extracting lesion information from these data is only realistic if automatic segmentation algorithms are not confined to multispectral images. Therefore, the development of a robust automatic lesion detection approach, which is capable of detecting lesions from a single anatomical MRI scan, is essential. A few attempts have been made to detect brain abnormality using T1-weighted MR images only. Cuadra et al. [35] used a priori models of lesion growth to segment large brain tumors. However, it is a semiautomatic approach where a seed voxel of tumors has to be chosen manually. The selection thus requires anatomical and biological knowledge of tumor growth. Stamatakis and Tyler [36] presented a statistical method to identify brain lesions. They compared individual patient images to a normal control group based on the general linear model and the detected significant structure differences between the patient and the controls were regarded as lesions. Srivastava et al. [37] employed a similar statistical approach to detect focal cortical dysplastic (FCD) lesions from a lesion-specific feature map constructed by computing the ratio of cortical thickness over absolute image intensity gradient. However, the disadvantage of these statistical segmentation methods is that the selection of a control group (e.g., group size) may have an impact on segmentation results [38]. Another potential limitation of this type of methods is that patient data and control images should be scanned using the same scanner, coils, and identical parameters, otherwise additional bias may be introduced. This issue hence restricts the general application of this type of methods to lesion identification.

As stated before, using the traditional segmentation method alone is insufficient to identify lesions from normal tissues owing to their intensity similarity. Among the studies using multispectral MR images, Wei et al. [39] demonstrated that the addition of a template-driven approach to EM algorithm significantly improved the segmentation accuracy of WM abnormalities. Kamber et al. [40] generated a 3-D tissue probability model using healthy controls and demonstrated that, with the assistance of the model, four segmentation methods tested reduced false positive detection of lesions by $50-80 \%$. These studies demonstrated that the usage of spatial information would improve the capability of traditional segmentation methods in lesion identification. It thus brings an idea that a proper combination of a traditional segmentation method and some spatial information of brain tissues may allow detecting lesions from a single anatomical MR image effectively.

Therefore, the main objective of this paper is to present a fully automatic 3-D segmentation approach to detect infarct lesions from a single anatomical MRI scan. It is implemented by calculating the likelihood of an inconsistency between segmentation results derived from voxel intensities and tissue partitions based on spatial location. More specifically, with a prior knowledge of normal tissue distribution in the brain, the probability of each voxel belonging to each tissue type is known in an MR image. If the same image is segmented based on voxel intensity, the voxels, where lesions are present, are more likely to be assigned to a different tissue class rather than the one suggested by the tissue distribution. Therefore, the degree of the inconsistency between them is calculated, which may indicate the likelihood of these voxels being lesions. In this paper, the conventional fuzzy c-mean (FCM) algorithm is employed for intensity-based segmentation. The spatial-location-based tissue distribution is provided by prior tissue probability maps.

In Section II, materials and the basic theory of FCM is introduced, as well as how the FCM algorithm cooperates with the tissue probability maps to detect lesions. In Section III, in order to validate the performance of our method quantitatively, simulated lesions controlled for variables such as lesion sizes and lesion signal reduction are presented, along with the definitions of performance evaluation parameters. Segmentation results on both simulated and real lesions are described in Section IV, in which the proposed method is also compared with the statistical lesion detection approach. Section V discusses issues relating to the detection accuracy of the proposed method. Conclusion is addressed in Section VI.

\section{MATERIAL AND METHODS}

\section{A. Image Acquisition}

In all, 15 stroke patients ${ }^{1}$ (female $=5$, mean age $=54.27$, and standard deviation $=10.96,>12$ months after stroke, seizurefree, and had no history of mental health problems) and 26 healthy subjects (male $=6$, mean age $=22.77$, and standard deviation = 4.47) have been scanned in this study. All MR images were acquired on a 3T Siemens Trio scanner (Erlangen, Germany). High-resolution 3-D brain MRI images were obtained using a T1-weighted magnetization prepared rapid acquisition gradient echo (MPRAGE) pulse sequence, with the following characteristics: repetition time $(\mathrm{TR})=1830 \mathrm{~ms}$, echo time $(\mathrm{TE})=4.43 \mathrm{~ms}$, inversion time $=1100 \mathrm{~ms}$, one acquisition, flip angle $=11^{\circ}$, field of view (FOV) $=256 \mathrm{~mm}, 176$ slices, voxel size $=1 \mathrm{~mm} \times 1 \mathrm{~mm} \times 1 \mathrm{~mm}$, and in-plane matrix = $256 \times 256$. Prior to scanning, all participants gave written informed consent according to the guidelines of the University of Surrey Ethical Review Board. Participants were paid for participation.

\section{B. FCM Clustering Algorithm}

The FCM clustering algorithm was introduced by Bezdek [41], which is one of the most popular segmentation methods in the literature [16], [18], [19]. When FCM is applied to the segmentation of gray-scale images, such as MRI, it classifies voxels in an image into a prespecified number of clusters based on voxel intensities. It employs a fuzzy membership $U$ to describe the degree of likeness of one voxel to each cluster. The membership is determined by intensity differences between the

\footnotetext{
${ }^{1}$ The stroke patients in this study who have either left- or right-hand motor deficits were recruited for a two-week recovery therapy.
} 
voxel and the center of each cluster, which is expressed as

$$
\mathbf{u}_{i j}=\frac{1}{\sum_{k=1}^{C}\left(\frac{d\left(\mathbf{x}_{j}, \mathbf{v}_{i}\right)}{d\left(\mathbf{x}_{j}, \mathbf{v}_{k}\right)}\right)^{2 /(m-1)}}
$$

where $\mathbf{x}_{j}(j=1, \ldots, N)$ represents the voxel intensity, $N$ is the number of voxels in an image, $\mathbf{v}_{i}(i=1, \ldots, C)$ refers to cluster center that is an approximation of average intensity in this cluster, while $C$ is the number of clusters, $d^{2}\left(\mathbf{x}_{j}, \mathbf{v}_{i}\right)$ measures a similarity between $\mathbf{x}_{j}$ and $\mathbf{v}_{i}$ by calculating their Euclidean distance, and $m \in(1, \infty)$ is a weighting exponent on each fuzzy membership, which controls the degree of fuzziness.

The FCM algorithm iteratively optimizes an objective function with continuous updates of fuzzy memberships and cluster center, until a convergence is reached. The detailed description of FCM can be found in [19]. As only one type of MR images is input for segmentation in this paper, the fuzzy membership $U$ is a $C \times N$ data matrix.

\section{Segmentation of Lesion Cluster}

Tissue probability maps are maps that describe the probability of each voxel belonging to specific classes of tissues in a standard space. They are usually created by images obtained from a large group of healthy participants, thus representing an averaged topology of tissues in the brain. The tissue probability maps used in this paper are modified versions of International Consortium for Brain Mapping (ICBM: http://www.loni.ucla. edu/ICBM/), which are the default maps provided in Statistical Parametric Mapping (SPM5: http://www.fil.ion.ucl.ac. $\mathrm{uk} / \mathrm{spm} /$ ). The original data were derived from $452 \mathrm{~T} 1$-weighted MR images, and then affine registered to the Montreal Neurological Institute (MNI) space and down sampled to $2 \mathrm{~mm}$ resolution.

Let $T$ be tissue probability maps, where $T$ is a $C \times N$ data matrix, and $C$ represents the number of probability maps, which is identical to the cluster number in FCM, and $\mathbf{t}_{i j} \subseteq T$ refers to the probability that the $i$ th voxel belongs to the $j$ th cluster.

The FCM algorithm segments each voxel into a prespecified number of tissues based on its intensity, while the tissue probability maps classify each voxel into these clusters of tissues according to the location of the voxel. Given an image from a healthy participant, the intensity-based and the location-based segmentation should be similar. However, if there are lesions present, the earlier two are more likely unmatched. Therefore, lesions may be detected by their extent of inconsistency. The higher the inconsistency at each voxel, the more likely it is a lesion.

$\mathbf{I}=\left\{\mathbf{I}_{1}, \mathbf{I}_{2}, \ldots, \mathbf{I}_{j}, \ldots, \mathbf{I}_{N}\right\}$ describes a measurement of inconsistency between the fuzzy membership and the probability maps. $\mathbf{I}_{j} \in[0,1]$. It is defined as

$\mathbf{I}_{j}= \begin{cases}0, & k=s \\ 1, & \mathbf{t}_{k j}<0.1 \text { and } k \neq s \\ \frac{\alpha \times\left|\mathbf{u}_{k j}-\mathbf{t}_{k j}\right|+\beta \times\left|\mathbf{t}_{s j}-\mathbf{u}_{s j}\right|,}{2} & \text { otherwise }\end{cases}$

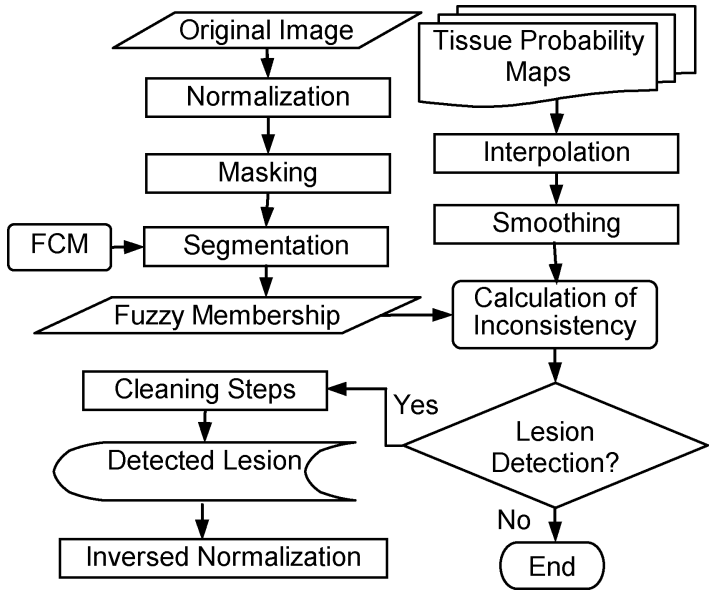

Fig. 1. Schematic diagram of segmentation procedure.

where $\alpha$ and $\beta$ are the weighting parameter $(\alpha \geq 1, \beta \geq 1)$, $\max \left(U_{j}\right)=\mathbf{u}_{k j}$ ( $k$ is the cluster with the highest membership at the $j$ th voxel), and $\max \left(T_{j}\right)=\mathbf{t}_{s j}$ ( $s$ is the cluster with the highest probability at the $j$ th voxel).

$$
\text { If } \mathbf{I}_{j}>\frac{\mathbf{u}_{k j}+\mathbf{t}_{s j}}{2} \text {, then the } j \text { th voxel }=\operatorname{lesion}(j=1, \ldots, N) \text {. }
$$

In more details, if the fuzzy membership and the tissue probability maps assign a voxel into the same cluster, then it is clear that they show no inconsistency $\left(\mathbf{I}_{j}=0\right)$. If the membership classifies the $j$ th voxel to the $k$ th cluster, the probability maps indicate that this voxel only has less than $10 \%\left(\mathbf{t}_{k j}<0.1\right)$ probability to be the same cluster, the inconsistency is defined as the maximum $\left(\mathbf{I}_{j}=1\right)$. In other cases, the measurement of inconsistency is derived from an average disagreement between the fuzzy membership and the probability, i.e., the difference between the maximum membership and the corresponding probability, plus the difference between the maximum probability and the corresponding membership. Weighting parameters $\alpha$ and $\beta$ are used to adjust the proportion of these two differences in the average. Finally, if the inconsistency at any voxel is higher than the mean of its maximum membership and probability, this voxel is then regarded as lesions.

\section{Segmentation Procedure}

Four probability maps are utilized in this approach (i.e., $C=4$ ), including background, CSF, GM, and WM, in which the background map is the inversed image of a brain mask, provided in SPM5 while the latter three maps are adapted versions of ICBM atlas.

Fig. 1 shows a schematic diagram of the whole segmentation procedure. As stated before, the probability maps are in the MNI space. In order to match the individual brain to the maps, an initial registration step is required to normalize original images to the MNI space. The normalization method employed is a unified segmentation approach embedded in SPM5, which enables image registration, tissue classification, and bias correction to be 
combined within the same generative model [21]. This approach can segment the healthy brains but provides no option of lesion detection. Likewise, the traditional normalization approach in SPM5 can also be used for this purpose. Depending on the voxel size of normalized MR images, the tissue probability maps are sampled to the identical resolution (in this paper: $1 \mathrm{~mm} \times$ $1 \mathrm{~mm} \times 1 \mathrm{~mm}$ ) using the trilinear interpolation algorithm. A smoothing kernel of full-width at half-maximum (FWHM) $=$ $10 \mathrm{~mm}$ is applied to the sampled maps so as to cover more varieties of the brain, such as different brain sizes and bigger/smaller ventricles.

The brain mask mentioned before is applied to normalized MR images for the removal of nonbrain regions, such as skull. Then, the brain-only images are segmented using the FCM algorithm into background, CSF, GM, and WM. The fuzzy memberships for each cluster are smoothed with a Gaussian kernel of $4 \mathrm{~mm}$ to increase the connectivity among neighboring voxels.

Next, the inconsistency between the fuzzy membership and the sampled and smoothed prior probability maps are calculated based on (2). The lesions are then identified by (3).

Small clusters are always present in detected lesions. They are more likely noise or artifacts rather than true lesions. Therefore, clusters with voxel numbers less than 1000 (i.e., volume of $1 \mathrm{~cm}^{3}$ ) are removed by morphological labeling. Furthermore, false positive segmentation happens often on edges of the brain because of residual variances to the probability maps after normalization. Hence, the detection of lesions excludes regions around edges of the brain using the brain mask. Finally, lesions are inversely normalized from the MNI space back to the original brain space.

\section{VALIDATION}

\section{A. Simulated Lesion}

In order to validate the proposed lesion detection method quantitatively, simulated lesions were introduced into images from healthy participants. Therefore, exact sizes and locations of lesions were known, which could be regarded as the ground truth of lesions. Instead of using sphere or cube, irregular shapes of simulated lesions, which are more representative to real lesions, were generated using a 3-D region of interests (ROI) creation tool provided in MRIcro (http://www.sph.sc.edu/comd/ rorden/mricro.html). Initially, origins of ROIs were chosen manually, along with varied radius and intensity differences at both origin and edge. The created 3-D ROIs were saved as binary masks and then overlaid on images of healthy participants individually. The intensities within the ROIs were reduced by a uniform percentage of original intensities in the overlaid images. According to analyses on real lesions, it was reported that lesioned areas in T1-weighted images comprise 30-90\% signal reduction when compared to healthy tissues [36]. Therefore, to cover the variety of lesions, each simulated lesion in this paper was constructed to include an intensity reduction of $20 \%$, $40 \%, 60 \%$, and $80 \%$, respectively. Nineteen ROIs in different sizes $\left(1.147-153.743 \mathrm{~cm}^{3}\right.$, mean $\left.=54.34 \mathrm{~cm}^{3}\right)$ and locations were generated. Hence, a total of $19 \times 4$ images with simulated lesions were created for validation purpose.

\section{B. Performance Evaluation Parameters}

To evaluate the performance of the lesion detection method, three popular accuracy measurements were used, introduced by [24]

$$
\begin{aligned}
\text { sensitivity } & =\frac{A_{\mathrm{ref}} \cap A_{\mathrm{seg}}}{A_{\mathrm{ref}}} \\
\text { specificity } & =\frac{\left(I-A_{\mathrm{ref}}\right) \cap\left(I-A_{\mathrm{seg}}\right)}{I-A_{\mathrm{ref}}} \\
\text { similarity index } & =2 \times \frac{A_{\mathrm{ref}} \cap A_{\mathrm{seg}}}{A_{\mathrm{ref}} \cup A_{\mathrm{seg}}}
\end{aligned}
$$

where $A_{\text {ref }}$ represents the ground truth, which is regions with true lesions (i.e., simulated lesions in this paper). $A_{\text {seg }}$ represents detected lesions by the proposed method. $I$ refers to the whole image for segmentation.

Sensitivity measures a true positive detection rate, and specificity measures a true negative detection rate. Similarity index (SI) is derived from a reliability measure known as the kappa statistic, which is sensitive to both size and location of segmentation [23], [24], [31], [42], [43].

\section{RESUlts}

In this section, experimental results are described to validate the performance of the proposed method. Both simulated and real lesions were employed. With the simulated lesions, the detection accuracy was measured using the evaluation parameters defined before. The real infarct lesions from stroke patients were also used to demonstrate the capability of the proposed method in practice. The results of a comparison between our method and a statistical approach presented in [36] were described.

Unless otherwise stated, a value of 2 was employed for the weighting exponent $\mathrm{m}$ in FCM, since it yielded the best results in most images and generally used in the application of FCM. Additionally, $\alpha=1.5$ and $\beta=1$ were used in (2). The whole lesion detection approach was implemented in MATLAB. It took approximately $4 \mathrm{~min}$ to process an image on a Window XP computer with $2.80 \mathrm{GHz}$ CPU and $3.5 \mathrm{~GB}$ of RAM.

\section{A. Simulated Lesion}

When images with simulated lesions were segmented, the results suggested that the lesions were generally well detected. Fig. 2 illustrates four examples of the detected results at the simulated lesions with $60 \%$ of intensity reduction. Fig. 3 shows a bar chart of the average sensitivity, specificity, and SI at different intensity reduction rates. It demonstrated that the highest accuracy was achieved at the lesions with $60 \%$ intensity reduction (sensitivity $=0.900$, specificity $=0.999, \mathrm{SI}=0.879$ ), while with the $20 \%$ reduction, the accuracy is the lowest (sensitivity = 0.385 , specificity $=0.999, \mathrm{SI}=0.511$ ).

Fig. 4 shows the sensitivity and SI with regards to the lesion volume. It is clear that with a higher intensity reduction in lesioned area, segmentation accuracies were not affected by lesion volume. In contrast, with a lower intensity reduction, segmentation accuracies varied but with no relation to lesion 


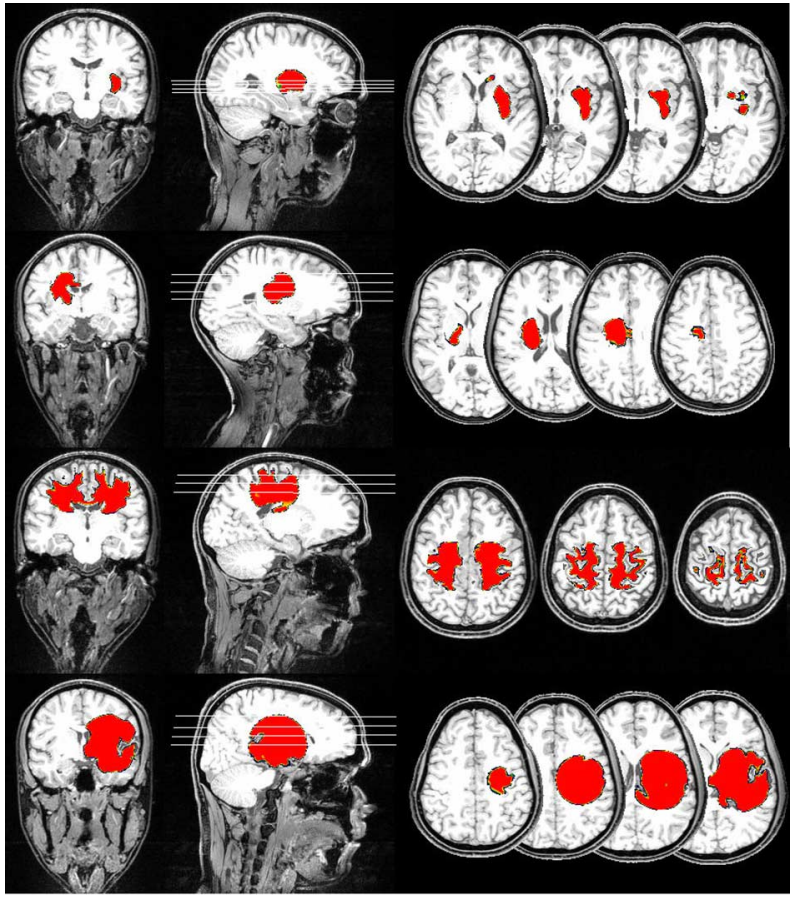

Fig. 2. Detected lesion on four simulated images. Lesion volume: $7.472 \mathrm{~cm}^{3}$ (first row), $13.693 \mathrm{~cm}^{3}$ (second row), $81.410 \mathrm{~cm}^{3}$ (third row), and $145.691 \mathrm{~cm}^{3}$ (fourth row). Each row includes coronal, sagittal, and axial views of each simulated image (from left to right).

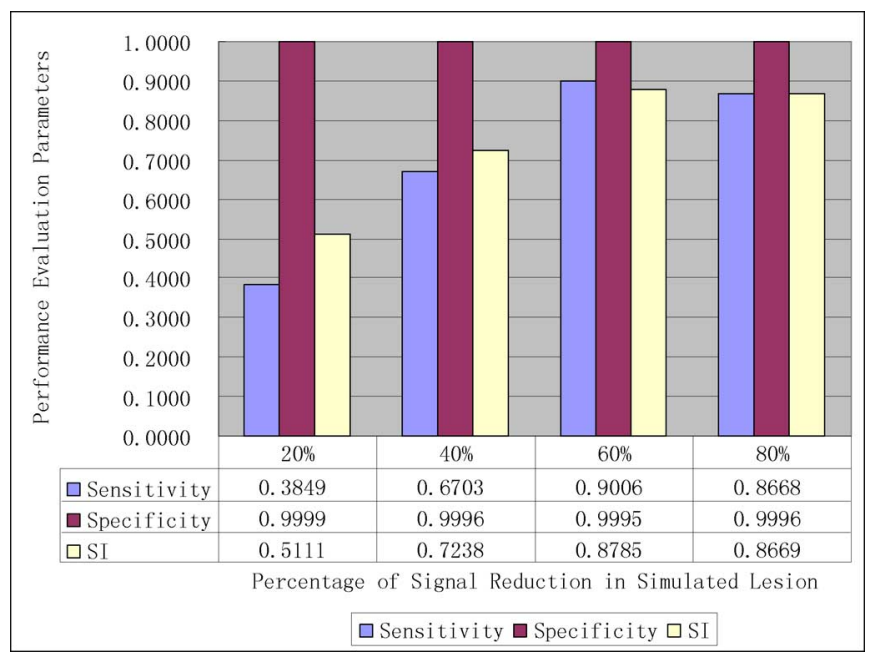

Fig. 3. Bar chart of performance evaluation parameters of lesion detection at different percentage of intensity reduction in simulated lesion area.

volume. As the specificity was greater than 0.99 in all cases, it is therefore not shown in the figure with regards to lesion volume.

\section{B. Real Lesion}

The performance of the proposed method was also demonstrated on real lesioned images. Fig. 5 shows images from four different patients. The top row of images in each panel shows original T1-weighted patient images while the bottom


Fig. 4. Sensitivity and SI with lesion volume.

row shows detected lesions superimposed on the same MRI scans.

In the 15 images tested, the detected infarct lesions in both GM and WM were in broad agreement with the real lesions based on visual inspection. The enlargement of ventricle was also identified. In some cases, false positive detection occurred around the ventricle, such as patient 3 [Fig. 5(c)], where the anterior part of left ventricle was labeled as lesion. Furthermore, lesions on cortical regions were sometimes undetected such as in patient 4 (left hemisphere).

\section{Comparison Between the Proposed Method and the Statistical Lesion Detection Method}

The statistical lesion detection method described in [36] and [37] was tested to compare with the proposed method. The control group included 26 healthy participants. Individual stroke patients were compared to the whole control group, after the normalization of all images to the MNI space and smoothing by a Gaussian kernel of FWHM $=8 \mathrm{~mm}$. Brain volume, age, and gender were included in the design matrix to exclude confounding effects. Significant structure differences (i.e., lesions) between each patient and the control group were detected using a threshold of $p<0.001$ (uncorrected). The parameters employed, such as the smoothing kernel and the threshold, were suggested to be optimal for lesion detection in terms of [36].

Overall, the statistical method acquired smoother regions of lesions, but its performance was not always consistent. Fig. 6 shows three examples of results in the normalized (MNI) space obtained from both the statistical and the proposed methods. Fig. 6(a) illustrates the detections on patient 5, where lesions 


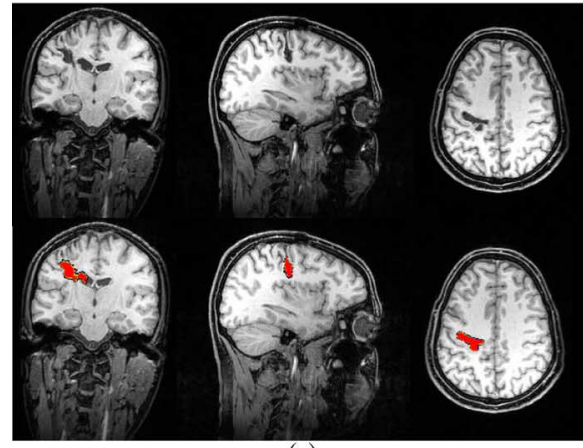

(a)

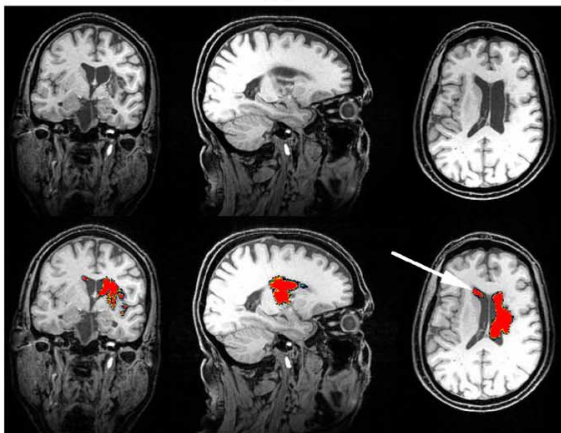

(c)



(b)

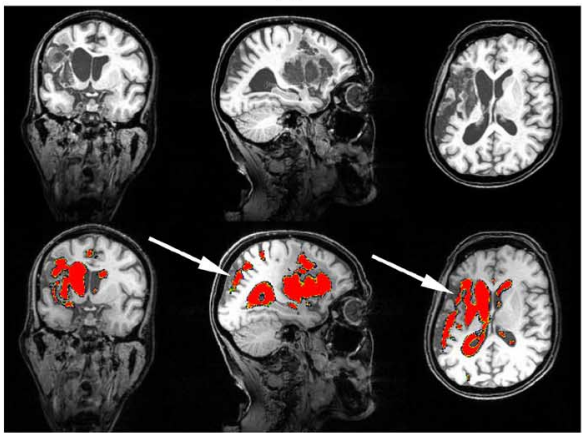

(d)

Fig. 5. Four examples of lesion detection on images from stroke patients. Top panel of each patient shows the original T1-weighted image. Bottom panel of each patient shows the lesions detected by the method proposed. (a) Patient 1. (b) Patient 2. (c) Patient 3. (d) Patient 4. Arrows point out the possible errors in lesion detection.
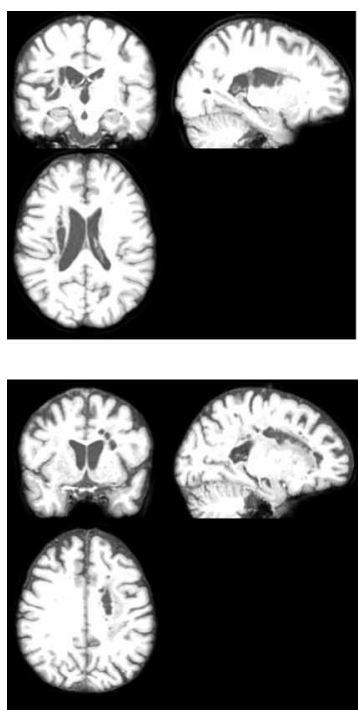

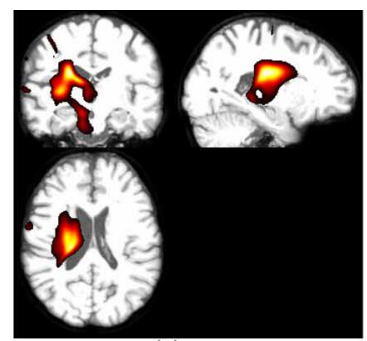

(a)


(b)


statistical method; right: lesion detected using the proposed method. (a) Patient 5. (b) Patient 6.

detected by the statistical method included false positive detection while the proposed segmentation method identified lesions more properly. Fig. 6(b) demonstrates that the statistical method was unable to identify lesions in patient 6 .

\section{DISCUSSION}

This paper presents a new lesion detection approach that only requires one type of anatomical MR image. The performance of the proposed method was tested on both simulated and real lesions. The use of simulated lesions provided the ground truth of lesion information, such as lesion size and location, which allowed us to validate the detection performance quantitatively. Different signal reduction percentages were introduced in lesioned areas. The results showed that simulated lesions with $20 \%$ signal reduction were detected with the lowest accuracy, while lesions with $60 \%$ lesions were detected with most precision. According to previous research in [36], in real lesions, 
the intensity near the lesion border, which consists of a mixture of the normal tissue and the lesion, is reduced less (30-40\%) as compared to the center of the lesion. As it is generally accepted that an SI greater than 0.7 indicates excellent agreement between detected lesions and true lesions [31], [42], [44], it is suggested that lesions with $40-80 \%$ intensity reduction were all well detected by the proposed method. This implied that our method was capable of identifying the major part of real lesions, with comparable accuracy to existing lesion detection methods using multispectral MRI scans [22]-[24], [43]. However, parts of lesion borders might be undetected if only minor lesions were included. In addition, it is worth noticing that the specificity acquired in our method is always higher than 0.99, because nonlesioned regions are much bigger than the region of lesions in each image.

When spherical simulated lesions were employed, the current method obtained an average of 0.96 for sensitivity and 0.99 for specificity, along with 0.97 for SI, which were better than the results of Stamatakis and Tyler [36]. [The highest true positive rate (sensitivity) and true negative rate (specificity) achieved were greater than 0.9.]

Real infarct lesions from stroke patients were detected using both the proposed method and the statistical approach presented in [36]. The results showed that the lesions were generally well detected by our method. The compared statistical approach identified lesions successfully in some images, but failed in others (as shown in Fig. 6). As stated in the literature [38], the selection of a control group (e.g., group size) may have an impact on segmentation results, while many covariates such as brain volume, age, and gender may also influence structure differences between a patient and a control group, thus altering the detection accuracy for lesions. All these factors are possible contributors to the inconsistent performance seen with the statistical lesion detection approach. It may be the case that if a higher number of healthy participants are included, the reliability of the statistical detection approach will be improved. However, further testing is required to comment on this issue.

Furthermore, in terms of the basic theory of the proposed method in this paper, the detection errors mainly occur due to a location mismatch between the fuzzy memberships and the tissue probability maps. Adjusting the settings and parameters involved may improve its detection performance. This includes reducing the false positive and false negative detection rates. More detailed discussion on these issues now follows.

\section{A. False Positive Detection}

False positive detection may occur under the following circumstances: first, when the maximum inconsistency is assigned, there is still a small possibility [although less than $10 \%$, $\mathbf{t}_{k j}<0.1$ in (2)] that the tissue probability indicates a segmentation identical to the membership. In order to lower the false positive rate due to the aforementioned reason, the percentage may be reduced.

Second, false positive detection may also be found around the ventricle because of its varied shape and size across participants. Increasing the smoothing kernel size used for tissue probability maps loosens constraints on spatial location, and thus, may reduce false positive detection in this region. Furthermore, an enlargement of ventricles was identified as lesions in the current approach, but it might be considered as false positive in some applications. To prevent ventricle detection, a CSF template could be applied.

\section{B. False Negative Detection}

As stated in the results, lesions in cortical regions may be partly undetected. In fact, cortical lesions were identified in most cases, but then were eliminated from the final detection by the brain mask in the cleaning step. However, without applying the mask, a great deal of false positive detections may be included in the results. This is due to residual errors of normalization between the normalized brain and the tissue probability maps. To solve this problem, either the mask could not to be applied or the threshold of masking could be reduced.

In addition, small clusters with less than 1000 voxels $\left(1 \mathrm{~cm}^{3}\right.$ in 3-D volume) were removed from the final detection by the cleaning step. Although a cluster smaller than this size is likely to be noise, especially for an infarct lesion, true lesions may still be removed. Hence, where there are small lesions, the threshold of 1000 voxels could be lowered.

Moreover, an increase of weighting parameters in (2) may enhance the sensitivity (reduce the false negative) of detection but lower the specificity, and vice versa. This is clear from the equation that higher $\alpha$ and $\beta$ lead to a greater inconsistency and raise the likelihood of a brain region being detected as lesions.

Most importantly, it is worth noticing that there is always a compromise between false positive and false negative detection. Reducing one often means increasing the other. Therefore, the settings of parameters may be adjusted in order to find the best balance according to specific characteristics of lesions studied.

\section{CONCLUSION}

An automated lesion detection method based on identifying inconsistent classification using voxel intensity and spatial location of tissues in MR images has been proposed in this paper. It requires only a single type of anatomical MR image, which is the most prominent advantage over other lesion detection methods.

The performance validation using simulated lesions suggested that the proposed method achieved detection accuracies highly comparable to other studies in which multispectral MR images were used. The identification of real infarct lesions from stroke patients further demonstrated the capability of the proposed method in lesion detection. Moreover, a statistical lesion detection approach, which also requires one single type of MR image, was applied to real lesions. The results when compared to those of the current method revealed that the current method is more reliable.

The disadvantages of the presented method include that small lesions $\left(<1 \mathrm{~cm}^{3}\right)$ are undetected due to the cleaning step after lesion detection. Additionally, the enlarged ventricle is always detected as a lesion that may be considered as false positive. Finally, parts of detected lesions around cortical regions may be 
removed by the application of the brain mask. Therefore, a bit of care is required when choosing the settings of the parameters.

According to characteristics of the proposed method, it may be capable of detecting lesions caused by brain injury, dementia, or tumor. Other MRI modalities, not restricted to T1-weighted images, may also be employed for the purpose of lesion detection. Finally, future work will focus on improving the current approach for a more general application, such as detecting small lesions, e.g., MS lesions.

\section{REFERENCES}

[1] The Stroke Association U.K. (2006, May). What is a stroke? [Online]. Available: http://www.stroke.org.uk/index.html

[2] J. Adamson, A. Beswick, and S. Ebrahim, "Is stroke the most common cause of disability?," J. Stroke Cerebrovasc. Dis., vol. 13, pp. 171-177, 2004.

[3] D. E. Saunders, A. G. Clifton, and M. M. Brown, "Measurement of infarct size using MRI predicts prognosis in middle cerebral artery infarction," Stroke, vol. 26, pp. 2272-2276, 1995.

[4] J. L. Saver, K. C. Johnston, D. Homer, R. Wityk, W. Koroshetz, L. L. Truskowski, and E. C. Haley, "Infarct volume as a surrogate or auxiliary outcome measure in ischemic stroke clinical trials," Stroke, vol. 30, pp. 293-298, 1999.

[5] R. Pineiro, S. T. Pendlebury, S. Smith, D. Flitney, A. M. Blamire, P. Styles, and P. M. Matthews, "Relating MRI changes to motor deficit after ischemic stroke by segmentation of functional motor pathways," Stroke, vol. 31, pp. 672-679, 2000.

[6] S. K. Schiemanck, M. W. M. Post, G. Kwakkel, T. D. Witkamp, L. J. Kappelle, and A. J. H. Prevo, "Ischemic lesion volume correlates with long-term functional outcome and quality of life of middle cerebral artery stroke survivors," Restorative Neurol. Neurosci., vol. 23, pp. 257-263, 2005.

[7] N. M. Menezes, H. Ay, M. W. Zhu, C. J. Lopez, A. B. Singhal, J. O. Karonen, H. J. Aronen, Y. Liu, J. Nuutinen, W. J. Koroshetz, and A. G. Sorensen, "The real estate factor: Quantifying the impact of infarct location on stroke severity," Stroke, vol. 38, pp. 194-197, 2007.

[8] J. Vock, P. Achermann, M. Bischof, M. Milanova, C. Muller, A. Nirkko, C. Roth, and C. L. Bassetti, "Evolution of sleep and sleep EEG after hemispheric stroke," J. Sleep Res., vol. 11, pp. 331-338, 2002.

[9] C. Muller, P. Achermann, M. Bischof, A. Nirkko, C. Roth, and C. Bassetti, "Visual and spectral analysis of sleep EEG in acute hemispheric stroke," Eur. Neurol., vol. 48, pp. 164-171, 2002.

[10] B. Yuan, G. J. Klir, and J. F. Swan-Stone, "Evolutionary fuzzy c-means clustering algorithm," in Proc. 1995 IEEE Int. Conf. Fuzzy Syst. Int. Joint Conf. 4th IEEE Int. Conf. Fuzzy Syst. 2nd Int. Fuzzy Eng. Symp., pp. 663670.

[11] T. Ogawa, A. Inugami, H. Fujita, J. Hatazawa, E. Shimosegawa, K. Noguchi, T. Okudera, I. Kanno, K. Uemura, and A. Suzuki, "MR diagnosis of subacute and chronic subarachnoid hemorrhage: Comparison with CT," Amer. J. Roentgenol., vol. 165, pp. 1257-1262, 1995.

[12] R. Adolphs, H. Damasio, D. Tranel, G. Cooper, and A. R. Damasio, "A role for somatosensory cortices in the visual recognition of emotion as revealed by three-dimensional lesion mapping," J. Neurosci., vol. 20, pp. 2683-2690, 2000.

[13] E. Bates, S. M. Wilson, A. P. Saygin, F. Dick, M. I. Sereno, R. T. Knight, and N. F. Dronkers, "Voxel-based lesion-symptom mapping," Nature Neurosci., vol. 6, pp. 448-450, 2003.

[14] G. H. L. Lemieux, K. Krakow, and F. G. Woermann, "Fast, accurate, and reproducible automatic segmentation of the brain in weighted volume MRI data," Magn. Reson. Med., vol. 42, pp. 127-135, 1999.

[15] H. Tang, E. X. Wu, Q. Y. Ma, D. Gallagher, G. M. Perera, and T. Zhuang, "MRI brain image segmentation by multi-resolution edge detection and region selection," Comput. Med. Imag. Graph., vol. 24, pp. 349-357, 2000.

[16] A. W. C. Liew and H. Yan, "An adaptive spatial fuzzy clustering algorithm for 3-D MR image segmentation," IEEE Trans. Med. Imag., vol. 22, no. 9, pp. 1063-1075, Sep. 2003.

[17] K. Van Leemput, F. Maes, D. Vandermeulen, and P. Suetens, "Automated model-based tissue classification of MR images of the brain," IEEE Trans. Med. Imag., vol. 18, no. 10, pp. 897-908, Oct. 1999.
[18] D. L. Pham and J. L. Prince, "Adaptive fuzzy segmentation of magnetic resonance images," IEEE Trans. Med. Imag., vol. 18, no. 9, pp. 737-752, Sep. 1999.

[19] S. Shen, W. Sandham, M. Granat, and A. Sterr, "MRI fuzzy segmentation of brain tissue using neighborhood attraction with neural-network optimization," IEEE Trans. Inf. Technol. Biomed., vol. 9, no. 3, pp. 459-467, Sep. 2005.

[20] D.-Q. Zhang and S.-C. Chen, "A novel kernelized fuzzy C-means algorithm with application in medical image segmentation," Artif. Intell. Med., Artif. Intell. Med. China, vol. 32, pp. 37-50, 2004.

[21] J. Ashburner and K. J. Friston, "Unified segmentation," NeuroImage, vol. 26, pp. 839-851, 2005.

[22] P. Anbeek, K. L. Vincken, M. J. P. van Osch, R. H. C. Bisschops, and J. Van Der Grond, "Automatic segmentation of different-sized white matter lesions by voxel probability estimation," Med. Image Anal., vol. 8, pp. 205-215, 2004.

[23] P. Anbeek, K. L. Vincken, M. J. P. van Osch, R. H. C. Bisschops, and J. Van Der Grond, "Probabilistic segmentation of white matter lesions in MR imaging," NeuroImage, vol. 21, pp. 1037-1044, 2004.

[24] P. Anbeek, K. L. Vincken, G. S. van Bochove, M. J. P. van Osch, and J. Van Der Grond, "Probabilistic segmentation of brain tissue in MR imaging," NeuroImage, vol. 27, pp. 795-804, 2005.

[25] B. R. Sajja, S. Datta, R. He, M. Mehta, R. K. Gupta, J. S. Wolinsky, and P. A. Narayana, "Unified approach for multiple sclerosis lesion segmentation on brain MRI," Ann. Biomed. Eng., vol. V34, pp. 142-151, 2006.

[26] S. Datta, B. R. Sajja, R. He, J. S. Wolinsky, R. K. Gupta, and P. A. Narayana, "Segmentation and quantification of black holes in multiple sclerosis," NeuroImage, vol. 29, pp. 467-474, 2006.

[27] K. Van Leemput, F. Maes, D. Vandermeulen, A. Colchester, and P. Suetens, "Automated segmentation of multiple sclerosis lesions by model outlier detection," IEEE Trans. Med. Imag., vol. 20, no. 8, pp. 677-688, Aug. 2001.

[28] F. B. Mohamed, S. Vinitski, C. F. Gonzalez, S. H. Faro, F. A. Lublin, R. Knobler, and J. E. Gutierrez, "Increased differentiation of intracranial white matter lesions by multispectral 3D-tissue segmentation: Preliminary results," Magn. Reson. Imag., vol. 19, pp. 207-218, 2001.

[29] A. Achiron, S. Gicquel, S. Miron, and M. Faibel, "Brain MRI lesion load quantification in multiple sclerosis: A comparison between automated multispectral and semi-automated thresholding computer-assisted techniques," Magn. Reson. Imag., vol. 20, pp. 713-720, 2002.

[30] A. B. B. Alfano, M. Larobina, M. Quarantelli, E. Tedeschi, A. Ciarmiello, E. M. Covelli, and M. Salvatore, "Automated segmentation and measurement of global white matter lesion volume in patients with multiple sclerosis," J. Magn. Reson. Imag., vol. 12, pp. 799-807, 2000.

[31] A. P. Zijdenbos, B. M. Dawant, R. A. Margolin, and A. C. Palmer, "Morphometric analysis of white matter lesions in MR images: Method and validation," IEEE Trans. Med. Imag., vol. 13, no. 4, pp. 716-724, Dec. 1994.

[32] J. K. Udupa, L. Wei, S. Samarasekera, Y. Miki, M. A. van Buchem, and R. I. Grossman, "Multiple sclerosis lesion quantification using fuzzyconnectedness principles," IEEE Trans. Med. Imag., vol. 16, no. 5, pp. 598-609, Oct. 1997.

[33] M. C. Clark, L. O. Hall, D. B. Goldgof, R. Velthuizen, F. R. Murtagh, and M. S. Silbiger, "Automatic tumor segmentation using knowledge-based techniques," IEEE Trans. Med. Imag., vol. 17, no. 2, pp. 187-201, Apr. 1998.

[34] F. Yang, T. Jiang, W. Zhu, and F. Kruggel, "White matter lesion segmentation from volumetric MR images," Lect. Notes Comput. Sci., vol. 3150 , pp. 113-120, 2004.

[35] M. B. Cuadra, C. Pollo, A. Bardera, O. Cuisenaire, J.-G. Villemure, and J.-P. Thiran, "Atlas-based segmentation of pathological MR brain images using a model of lesion growth," IEEE Trans. Med. Imag., vol. 23, no. 10, pp. 1301-1314, Oct. 2004

[36] E. A. Stamatakis and L. K. Tyler, "Identifying lesions on structural brain images-Validation of the method and application to neuropsychological patients," Brain Lang., vol. 94, pp. 167-177, 2005.

[37] S. Srivastava, F. Maes, D. Vandermeulen, W. V. Paesschen, P. Dupont, and P. Suetens, "Feature-based statistical analysis of structural MR data for automatic detection of focal cortical dysplastic lesions," NeuroImage, vol. 27, pp. 253-266, 2005.

[38] S. Shen, A. J. Szameitat, and A. Sterr, "VBM lesion detection depends on the normalization template: A study using simulated atrophy," Magn. Reson. Imag., vol. 25, pp. 1385-1396, 2007. 
[39] X. Wei, S. K. Warfield, K. H. Zou, Y. Wu, X. Li, A Guimond, J. P. Mugler, III, R. R. Benson, L. Wolfson, H. L. Weiner, and C. R. G. Guttmann, "Quantitative analysis of MRI signal abnormalities of brain white matter with high reproducibility and accuracy," J. Magn. Reson. Imag., vol. 15, pp. 203-209, 2002.

[40] M. Kamber, R. Shinghal, D. L. Collins, G. S. Francis, and A. C. Evans, "Model-based 3-D segmentation of multiple sclerosis lesions in magnetic resonance brain images," IEEE Trans. Med. Imag., vol. 14, no. 3, pp. 442453, Sep. 1995.

[41] J. C. Bezdek, Pattern Recognition With Fuzzy Object Function Algorithms. Norwell, MA: Kluwer, 1981.

[42] M. S. Atkins and B. T. Mackiewich, "Fully automatic segmentation of the brain in MRI," IEEE Trans. Med. Imag., vol. 17, no. 1, pp. 98-107, Feb. 1998.

[43] W. Li, J. Tian, E. Li, and J. Dai, "Robust unsupervised segmentation of infarct lesion from diffusion tensor MR images using multiscale statistical classification and partial volume voxel reclassification," NeuroImage, vol. 23, pp. 1507-1518, 2004.

[44] J. Bartko, "Measurement and reliability: Statistical thinking considerations," Schizophrenia Bull., vol. 17, pp. 483-489, 1991.

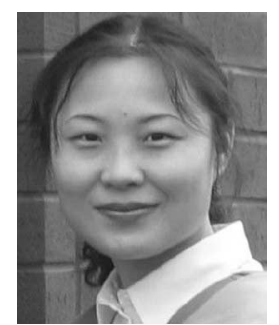

Shan Shen received the B.S. and M.S. degrees in communication engineering and biomedical engineering from the University of Chongqing, Chongqing, China, in 1997 and 2000, respectively, and the $\mathrm{Ph} . \mathrm{D}$. degree in electronic engineering and bioengineering from the University of Strathclyde, Glasgow, U.K., in 2004.

In 2004, she joined the Department of Psychology, University of Surrey, Guildford, U.K., where she is currently a Research Fellow. Her current research interests include structural and functional MR image analysis, medical imaging, genetic programming, and neural networks.

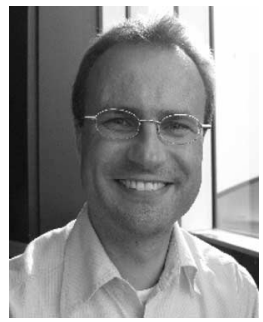

André J. Szameitat received the Diploma in psychology from the Technical University Braunschweig, Braunschweig, Germany, in 1999, and the Ph.D. degree psychology from the Max Planck Institute of Cognitive Neuroscience (now Max Planck Institute for Human Cognitive and Brain Science), Leipzig, Germany, in 2002.

He is currently a Research Fellow in the Department of Psychology, University of Surrey, Guildford, U.K. His current research interests include functional magnetic resonance imaging of the premotor and motor system and cognitive research into the control of actions.

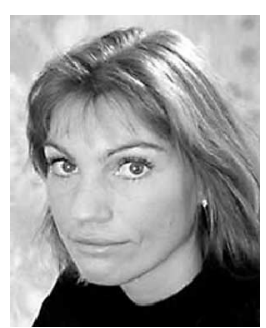

Annette Sterr received a degree in psychology and biology and the $\mathrm{Ph} . \mathrm{D}$. degree in psychology from the University of Konstanz, Konstanz, Germany, in 1995 and 1998, respectively.

During 1995-1996, she was a Research Fellow at the University of California, San Diego. From 2000 to 2003, she was a Lecturer/Reader with the University of Liverpool, where she established the Luria Laboratory for Clinical Neuroscience. She is currently the Chair in Cognitive Neuroscience and Neuropsychology, University of Surrey, Guildford, U.K. Her articles have been published in the highest ranking journals such as Nature, Journal of Neuroscience, and Neurology. Her current research interests include constraint-induced movement therapy, motor rehabilitation, neuropsychology of acquired brain injury, cognition, and functional brain organization.

Dr. Sterr received the Young Scientist Awards in Japan and Germany in 1997 and 1998. She was chosen by the Medical Research Council (MRC) as one of their Career Development Fellows in 2002. 